\title{
Multi-Tb/s sustainable MAN scenario enabled by VCSEL-based innovative technological solutions
}

\author{
Pierpaolo Boffi*a, Paola Parolari ${ }^{\mathrm{a}}$, Alberto Gatto ${ }^{\mathrm{a}}$, Mariangela Rapisarda ${ }^{\mathrm{a}}$, \\ Michela Svaluto Moreolo ${ }^{\mathrm{b}}$, Laia Nadal ${ }^{\mathrm{b}}$, Josep M. Fabrega ${ }^{\mathrm{b}}$, Nicola Calabretta ${ }^{\mathrm{c}}$, Ripalta Stabile ${ }^{\mathrm{c}}$, \\ Netsanet Tessema ${ }^{c}$, David Larrabeiti ${ }^{\mathrm{d}}$, Juan Pedro Fernàndez-Palacios ${ }^{\mathrm{e}}$, Gabriel Otero ${ }^{\mathrm{d}}$, \\ Christian Neumeyr ${ }^{\mathrm{f}}$, Giovanni Delrosso ${ }^{\mathrm{g}}$, S. Bhat ${ }^{\mathrm{g}}$, Karen Solis-Trapala ${ }^{\mathrm{h}}$, Giorgio Parladori ${ }^{\mathrm{i}}$ \\ aPolitecnico di Milano, Dip. Elettronica, Informazione e Bioingegneria, P.zza Leonardo da Vinci 32, \\ 20133, Milano, Italy; ${ }^{b}$ Centre Tecnològic de Telecomunicacions de Catalunya (CTTC/CERCA), \\ Castelldefels (Barcelona), Spain; ' Technische Universiteit Eindhoven (TU/e), Electrical Engineering \\ Department, Eindhoven, The Netherlands; ${ }^{\mathrm{d} U n i v e r s i d a d ~ C a r l o s ~ I I I ~ d e ~ M a d r i d, ~ M a d r i d, ~ S p a i n ; ~}$ \\ ${ }^{\mathrm{e}}$ Telefonica Global CTO, Madrid, Spain; fVertilas GmbH, Garching, Germany; ${ }^{\mathrm{g} V T T}$ Technical \\ Research Center of Finland, Espoo, Finland;

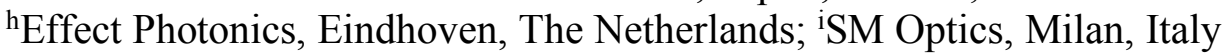 \\ *pierpaolo.boffi@polimi.it; phone +3902 23998923
}

\begin{abstract}
Innovative photonic solutions designed and developed in the H2020 research project PASSION are presented for the future metropolitan area network (MAN) supporting different aggregated data traffic volumes and operating at heterogenous granularities. System performance evaluated both by simulations and experimentation regarding the proposed vertical cavity surface emitting laser (VCSEL) -based modular sliceable bandwidth/bitrate variable transceiver (S-BVT) are shown in realistic MANs organized by hierarchical levels with the crossing of multiple nodes characterized by new switching/aggregation technologies. The capabilities and challenges of the proposed cost-effective, energy-efficient and reduced footprint technological solutions will be demonstrated to face the request of huge throughput and traffic scalability.
\end{abstract}

Keywords: Sliceable bandwidth/bitrate variable transceiver (S-BVT), vertical cavity surface emitting laser (VCSEL), metropolitan area network (MAN), discrete multitone (DMT) modulation, photonic technologies

\section{INTRODUCTION}

The future metropolitan area network (MAN) architecture [1] will support different aggregated data traffic volumes and operate at heterogenous granularities, assuring a huge total throughput in transmission and routing in order to support a large range of applications (including mobile transport in terms of backbone, backhaul, and fronthaul, data center interconnection and enterprise connectivity) and any kind of service (such as 5G, mobile edge computing, UHD TV, etc.). A net positive market CAGR of 5\% is expected till 2022 [2] for the metro optical equipment, which has to guarantee flexibility and scalability features, together with sustainability in terms of low cost, low power consumption and footprint. New photonic technologies are being explored to design alternative solutions compared to the traditional high-capacity approaches adopted for the long-haul fiber infrastructure, which mainly rely on ultra-high speed data rate per carrier [3] and on the exploitation of an ultra-wide band (S, C and L band) amplification [4]. Despite the use of silicon-photonics $(\mathrm{SiPh})$ devices and the support of hundreds of wavelength-division multiplexed (WDM) channels, in fact, these approaches still appear too expensive and power hungry for the future MAN.

To deal with the challenging requirements of the future agile MAN, an innovative photonic technological platform is designed in the framework of the EU-H2020 project PASSION [5], for the development of both a suitable modular transmitter able to assure multi-Tb/s capacity per photonic integrated circuit (PIC), and a switching node architecture featuring different levels of aggregation. In particular, the exploitation of long-wavelength high-bandwidth vertical cavity surface emitting laser (VCSEL) sources [6] directly modulated (DM) with discrete multitone (DMT) modulation [7] combined with multi-channel coherent receivers (CO-RXs) allows to realize a compact and cost-effective sliceable 
bandwidth and bitrate variable transceiver (S-BVT) [8] able to adaptively load/manipulate the spectrum, enabling tens of $\mathrm{Tb} / \mathrm{s}$ capacity per link exploiting multiple dimensions including the spectrum, the polarization and the space.

The paper is organized as follows. In Section 2 we describe the requests of the future MAN, showing its topology organized in hierarchical level (HL) nodes. Section 3 presents the C-band VCSEL technology based on a short cavity (SC) structure able to assure more than 50-Gb/s transmitted rate thanks to DMT. Section 4 shows in details the S-BVT architecture, whose building block is constituted by a 40-VCSEL module produced in silicon-photonics ( $\mathrm{SiPh}$ ) platform with massive integration on silicon-on-insulator (SOI) chips. Thanks to 50-Gb/s rate per DM VCSEL, up to 2-Tb/s capacity per module is obtained, while up to $16-\mathrm{Tb} / \mathrm{s}$ total capacity is aggregated by adopting a modular approach, matching the requirements for each hierarchical layer of the proposed MAN. In Section 5 the switching/aggregation node architecture is presented, which is equipped with different photonic fundamental modules, depending on the featured hierarchical levels and handling the added and dropped traffic in both space and spectrum dimensions. Finally, Section 6 shows the simulated performance of the DMT signal in a multichannel MAN characterized by numerous hops among the nodes and supported by CO-RXs, in order to evaluate the capabilities of the proposed VCSEL-based S-BVT. Conclusions are reported in Section 7.

\section{FUTURE MAN ORGANIZED BY HIERARCHICAL LEVELS}

As shown in Figure 1, we take in account a network layered topology [9] organized in HL nodes, from the HL1 routers on the top of the IP network interfacing the national backbone network (on the right), to HL4 nodes located at the bottom layer of the IP network and to HL5 nodes aggregating traffic from the users (on the left). HL2 nodes provide the access to MAN services, while HL3 nodes assure traffic aggregation/distribution function, collecting data traffic coming from different geographical MAN areas. Each HL node is connected to at least two adjacent nodes of the preceding hierarchical level, supporting different functionalities specific of its own HL.

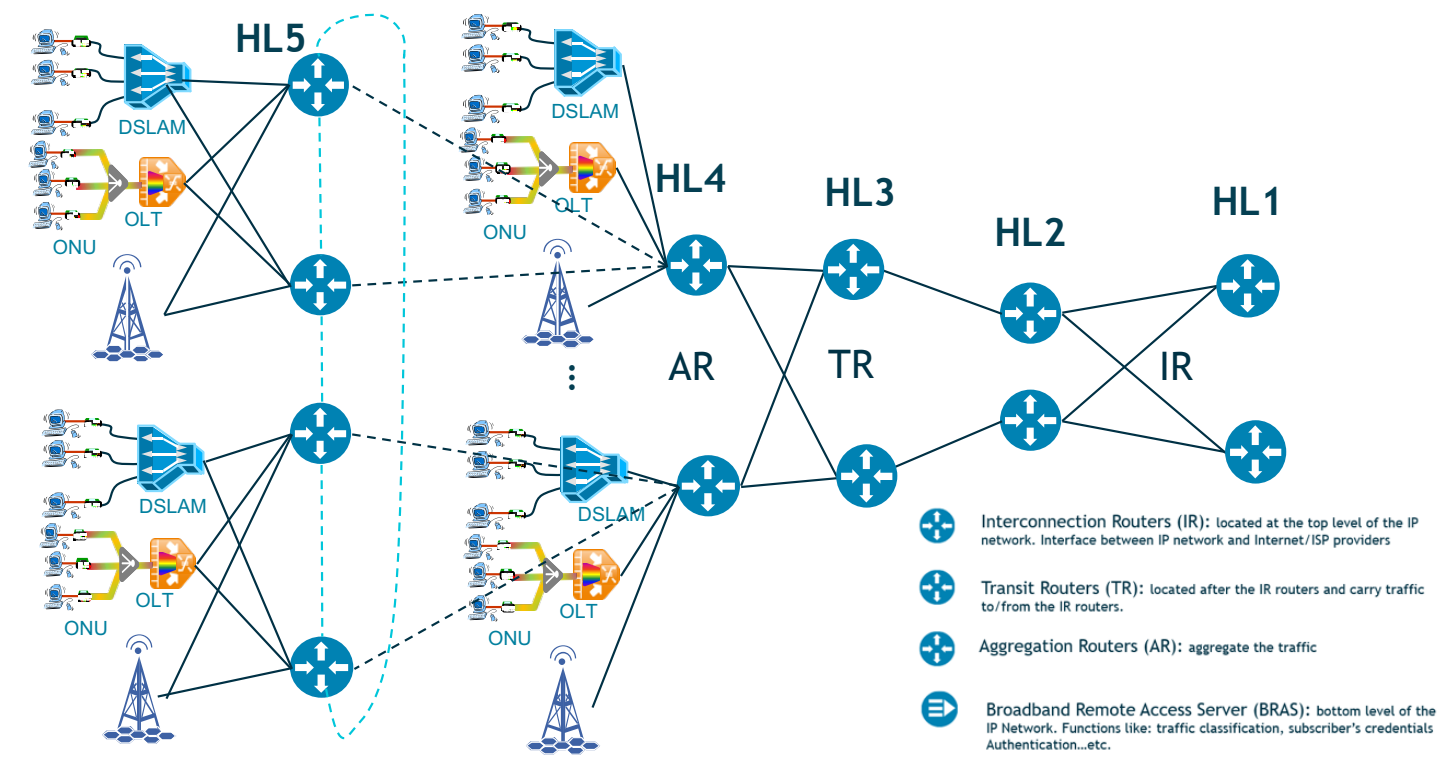

Figure 1. Schematic of the network layer hierarchy.

The future sustainable MAN scenario has to be designed considering the above architecture, with the request of costeffective ultra-broadband transport and expansion in a large area (also hundreds of km extended), targeting a feasible 'pay as you grow' scheme. Cheap upgradable optical sliceable transceivers have to guarantee at HL4 node up to 2-Tb/s rate, while at HL3 level dynamic capacity adaptation from $2 \mathrm{~Tb} / \mathrm{s}$ to $16 \mathrm{~Tb} / \mathrm{s}$ is expected, with higher capacity S-BVTs. Moreover, HL1/2 nodes have to be equipped with interfaces supporting aggregate capabilities of tens of Tb/s (e.g. $64 \mathrm{~Tb} / \mathrm{s}$ ).

For the design of the VCSEL-based S-BVT developed in PASSION project we have assumed to follow the hierarchical model and the requested capacities of the targeted MAN described above. In the following Sections, the details regarding the technological implementation are shown. 


\section{C-BAND DMT-MODULATED VCSEL FOR HIGH-CAPACITY S-BVT}

\subsection{Short cavity VCSELs}

Nowadays, GaAs VCSELs operating in the first window of fiber communications (around $850 \mathrm{~nm}$ ) are massively employed for intra-datacenter interconnections employing multi-mode fibers. VCSEL technology appears very promising in terms of cost, testability, and power consumption [10], but for its exploitation in applications such as MANs it is necessary to guarantee long-wavelength single-mode emission to limit the effect of modal and chromatic dispersion (CD) and to support dense WDM [11,12]. In PASSION project the company Vertilas GmbH has developed VCSEL sources in InP satisfying the above requirements [13]. Single-mode operation is achieved by an appropriate transverse waveguide structure with sidemode suppression ratio higher than $35 \mathrm{~dB}$ and a stable polarization output. C-band emission is assured by exploiting buried tunnel junction (BTJ) approach. The reduced active area allows an expected far field confined in less than $12^{\circ}$ with a structure optimized for massive integration. In order to achieve very high modulation rate in order to face the capacity demand of the considered MAN, in the developed VCSEL a short-cavity (SC) is obtained by a very short resonator length and optimized active region, guaranteeing modulation bandwidth beyond $14 \mathrm{GHz}$. Fig. 2 shows the SC VCSEL structure optimized for high modulation bandwidth (on the left) and the BTJ structure for C-band emission (on the right).

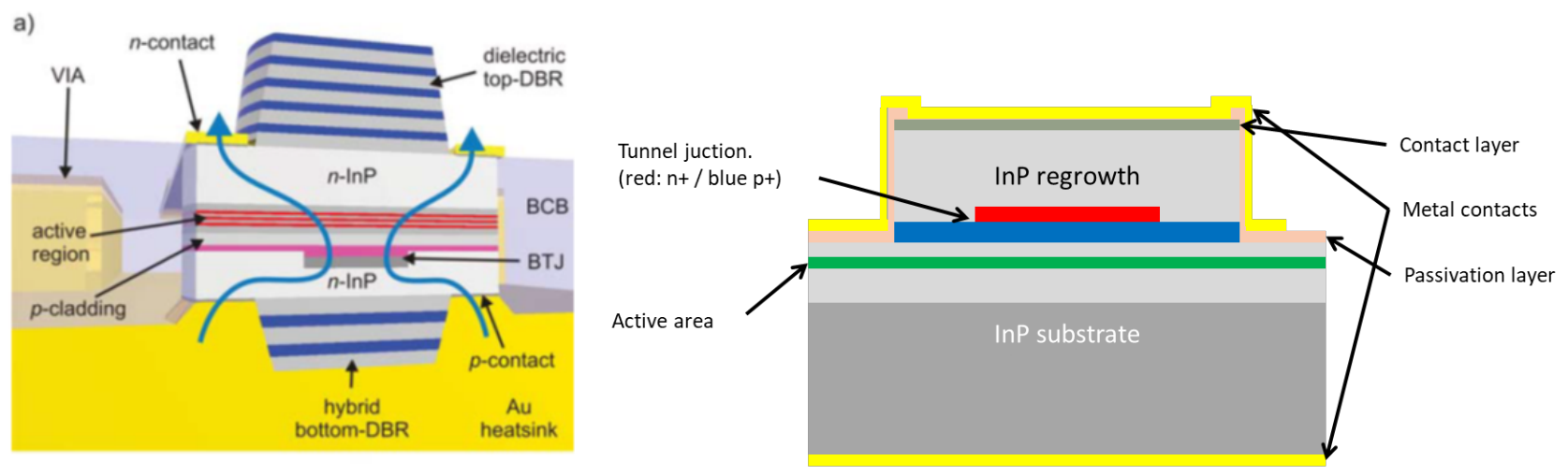

Figure 2. SC (left) and BTJ structure (right) of the developed InP VCSELs.

\subsection{DMT modulation and coherent detection}

Thanks to the developed features, more than $50-\mathrm{Gb} / \mathrm{s}$ rate per VCSEL are guaranteed also in case of DM by exploiting multi-carrier formats, such as DMT. As well known, DMT allows to increase the transported capacity, matching the nonuniform and bandwidth-limited response of the whole communication system [14,15] thanks to the exploitation of bit and power loading. In this way, DMT is capable to dynamically and flexibly adapt the VCSEL equivalent rate to traffic/channel conditions and spectrum fragmentation mitigation in the MAN [16], also as a function of the transmission path reach. 50$\mathrm{Gb} / \mathrm{s}$ modulation performance is achieved for the VCSEL combined to very low power consumption, less than $30 \mathrm{~mW}$. The main significant drawback of VCSEL DM remains the frequency chirp: for the developed InP SC VCSEL the measured chirp parameters correspond to a linewidth enhancement factor $\alpha$ of 3.7 and adiabatic constant $\kappa$ of $1.52 \cdot 10^{13}$ [17].

To achieve 25-GHz channel spacing in WDM multiplexing, while providing a higher resilience towards filtering effects, the dual sideband (DSB) spectrum of the VCSEL, modulated by the DMT signal, is filtered by properly detuning a 25GHz wavelength selective switching (WSS) filter, with 21-GHz full width half maximum (FWHM) [18]. We thus obtain a single sideband (SSB) spectrum, preserving the optical carrier, which is shown in Fig. 3 in case of a filter frequency detuning of about $9.5 \mathrm{GHz}$. The DMT signal is composed by 256 sub-carriers; in order to match the available VCSEL electro optic response, they cover a $16-20 \mathrm{GHz}$ range. A cyclic prefix of about $2.1 \%$ of the symbol length is also added. Chow's algorithm is used with measured signal to noise ratios (SNRs) to perform bit- and power-loading [19], setting the

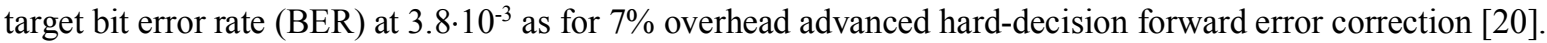


In order to target transmission distances typical of metro-regional networks, we employ a CO-RX, allowing CD compensation, avoiding also the VCSEL chirp interplay with CD. The chirp associated with VCSEL DM impairs transmission performance only due to the presence of filtering associated to wavelength multiplexers/demultiplexers and to nodes crossing. Due to DM, the transmitted signal is just intensity modulated and then a simplified digital signal processing (DSP) at the CO-RX side is exploited [20]: in fact, after I and Q components recovery and CD compensation, the I and Q square moduli are performed and summed up in order to obtain the originally transmitted intensity signal. Although this approach cancels the coherent detection advantages in terms of BER as a function of SNR, it avoids the use of phase and frequency recovery, relaxing the constraints on VCSEL and local oscillator (LO) linewidths. As shown in the Table of Fig. 3 right, this resilience of the reduced complexity CO-RX towards phase noise associated with the LO linewidth is verified by simulations. The Table reports the maximum capacity achieved in back to back condition in case of typical DM-VCSEL linewidth of $5 \mathrm{MHz}$, LO power of $10 \mathrm{dBm}$ per state of polarization (SOP), and 30-GHz photodiodes (PD) e/o bandwidth and noise equivalent current (NEC) of $20 \mathrm{pA} / \mathrm{Hz}^{1 / 2}$. The results obtained for $30 \mathrm{~dB}$ and $40 \mathrm{~dB}$ optical SNRs (OSNRs) confirm that the impact of the LO linewidth is almost negligible up to $1 \mathrm{MHz}$

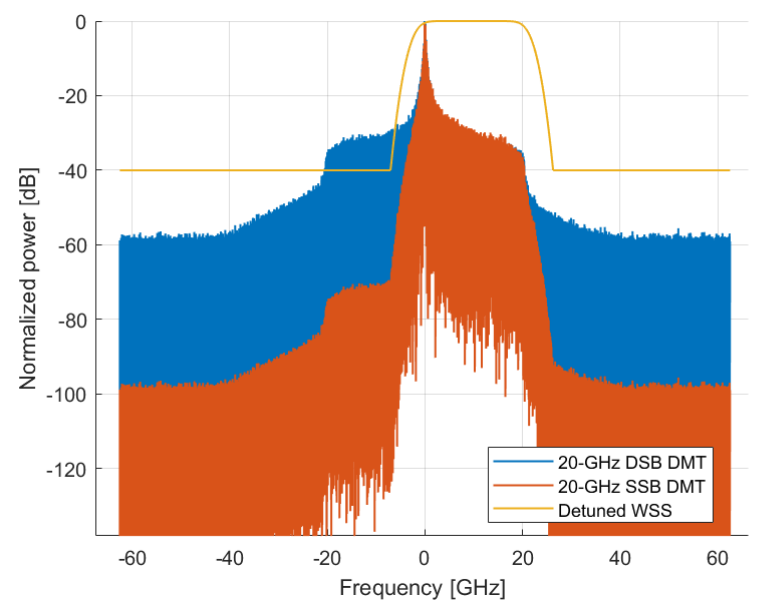

\begin{tabular}{|c|c|c|}
\hline OSNR & LO linewidth & Capacity \\
\hline \multirow{3}{*}{$\begin{array}{l}\hat{\theta} \\
\text { சै }\end{array}$} & $100 \mathrm{kHz}$ & $77 \mathrm{~Gb} / \mathrm{s}$ \\
\hline & $500 \mathrm{kHz}$ & $76.6 \mathrm{~Gb} / \mathrm{s}$ \\
\hline & $1 \mathrm{MHz}$ & $76.2 \mathrm{~Gb} / \mathrm{s}$ \\
\hline \multirow{3}{*}{$\stackrel{\oplus}{\vec{\theta}}$} & $100 \mathrm{kHz}$ & $58.1 \mathrm{~Gb} / \mathrm{s}$ \\
\hline & $500 \mathrm{kHz}$ & $57.7 \mathrm{~Gb} / \mathrm{s}$ \\
\hline & $1 \mathrm{MHz}$ & $57.3 \mathrm{~Gb} / \mathrm{s}$ \\
\hline
\end{tabular}

Fig. 3. Left: DMT spectra at the output of the DM VCSEL. DSB signal with 20-GHz electrical bandwidth (blue) and SSB (red) obtained by a 9.5-GHz detuned WSS (orange) filtering. Right: Table with SSB DMT capacity in back-to-back as a function of the LO linewidth, with respect to OSNR.

\section{MODULAR S-BVT FOR MULTI-Tb/s AGGREGATED CAPACITY}

The S-BVT developed within PASSION project is based on a 2-Tb/s module (MOD) with 40 VCSELs integrated in a silicon-on-insulator (SOI) platform [1]. As shown in Fig. 4 left, the square MOD contains 10 VCSELs with 400-GHz spacing on each side (sub-MOD). $50 \mathrm{~Gb} / \mathrm{s}$ rate is achieved per state of polarization (SOP) per VCSEL thanks to DMT, as described in Section 3. 4 arrayed waveguide grating (AWGs) multiplex the optical channels emitted from the 10 VCSELs in each sub-MOD, while a 2-stage Mach-Zehnder interferometer (MZ) multiplexer interleaves the 4 sub-MOD 10-channel outputs into a single waveguide transporting 40 multiplexed channels with 100-GHz granularity. In this way, the $4050-$ $\mathrm{Gb} / \mathrm{s}$ modulated VCSEL MOD demonstrates an aggregated capacity of up to $2 \mathrm{~Tb} / \mathrm{s}$, targeting the rate required for the HL4 layer. Fig. 4 right shows the realized SOI photonic integrated chip (PIC) of the MOD fabricated as part of the VTT MPW6 process run [21]. Integrated optical $45^{\circ}$ total internal reflective (TIR) mirrors on Si-PIC in up-reflecting configuration are employed for VCSEL Si waveguide coupling with high efficiency (coupling loss lower than 0.5dB). To electrically interconnect the SOI PIC with the 40 VCSEL sources, an electrical interposer based on a land grid array (LGA) pad architecture has been fabricated. Taking into account the channel pitch depending on the dimension of the VCSELs and the linear drivers, the achieved 2-Tb/s MOD chip size is about $4 \mathrm{~cm}^{2}$ with power consumption less than $5 \mathrm{pJ} / \mathrm{bit}$.

By combining 4 of such a 2-Tb/s MOD, a full 160-channels super-MOD is obtained, characterized by 25-GHz granularity covering the ITU C-band channels from CH19 (1562.23 nm) to CH59 (1530.33 nm), and enabling up to $8 \mathrm{~Tb} / \mathrm{s}$ total transmitted capacity (Fig. 4) per polarization. For the super-MOD we adopted a modular approach, allowing to design and develop identical MODs to build it: the 40 emitted wavelengths from the VCSELs in each MOD are fine-tuned in a range of $0-75 \mathrm{GHz}$ through the VCSEL bias current and stabilized by a temperature cooler. The adopted modularity offers the 
ability to fabricate and stock only one MOD type and to use the identical 40-channel MODs to build the full 160-channel TX super-MOD.

By employing CO-RX at the receiver side, polarization-division multiplexing (PDM) can be also exploited, coupling by means of a polarizing beam combiner (PBC) the outputs of 2 identical super-MODs, orthogonal in SOP, achieving up to $16 \mathrm{~Tb} / \mathrm{s}$ capacity. Coherent detection allows not only to demultiplex the SOPs, but also to compensate the chromatic dispersion in case of significant reaches, also hundreds of $\mathrm{km}$ long, typical of metro-regional networks.

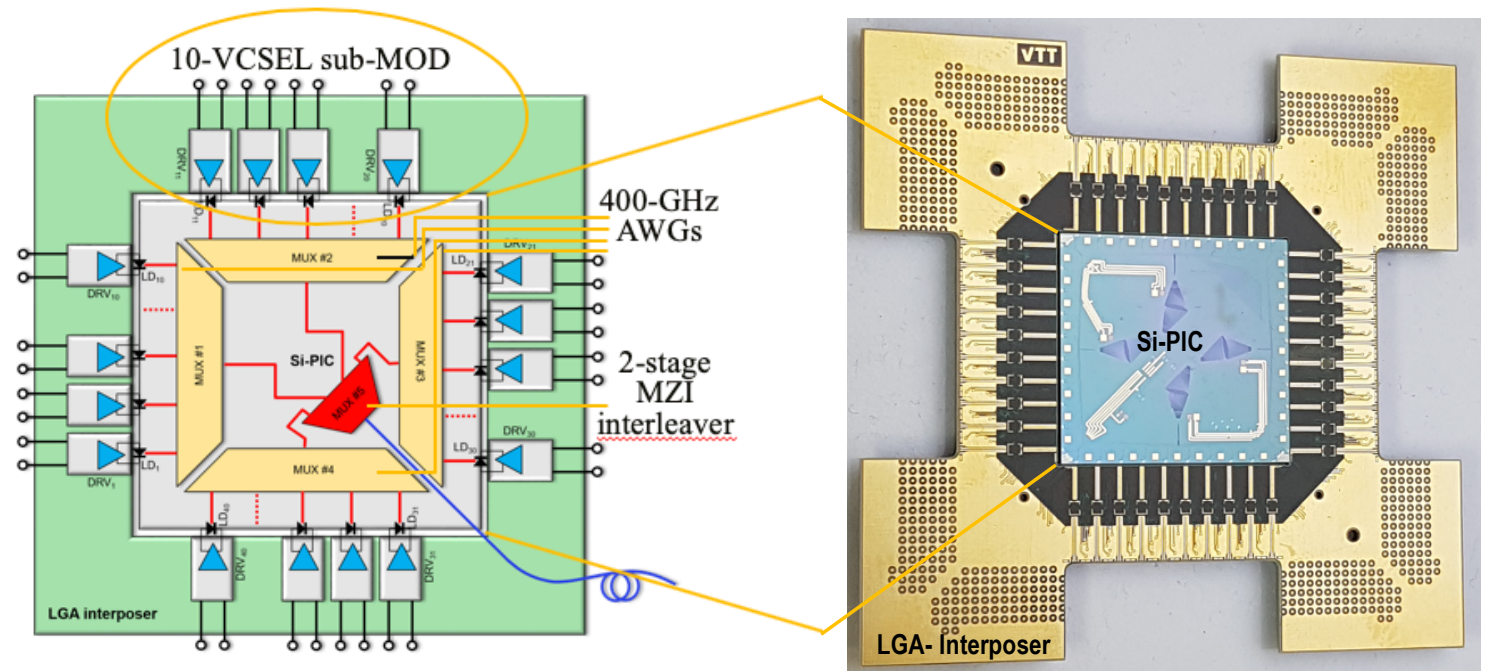

Fig. 4. Left: Scheme of the 40-VCSELs MOD providing a total capacity of 2-Tb/s. Right: realized SOI PIC of the MOD on the electrical interposer.

The developed modular S-BVT [22] can adaptively load/manipulate the spectrum with 25-GHz fine granularity, exploiting in a flexible way multicarrier modulation, the whole C-band spectrum and the polarization [23], matching all the requirements of the HL3 layer in terms of dynamic capacity adaptation. The employment of spatial multiplexing by means of bundles of fiber or multi-core fibers can allow to achieve an aggregate capacity of even tens of Tb/s supporting HL1/HL2 nodes demand.

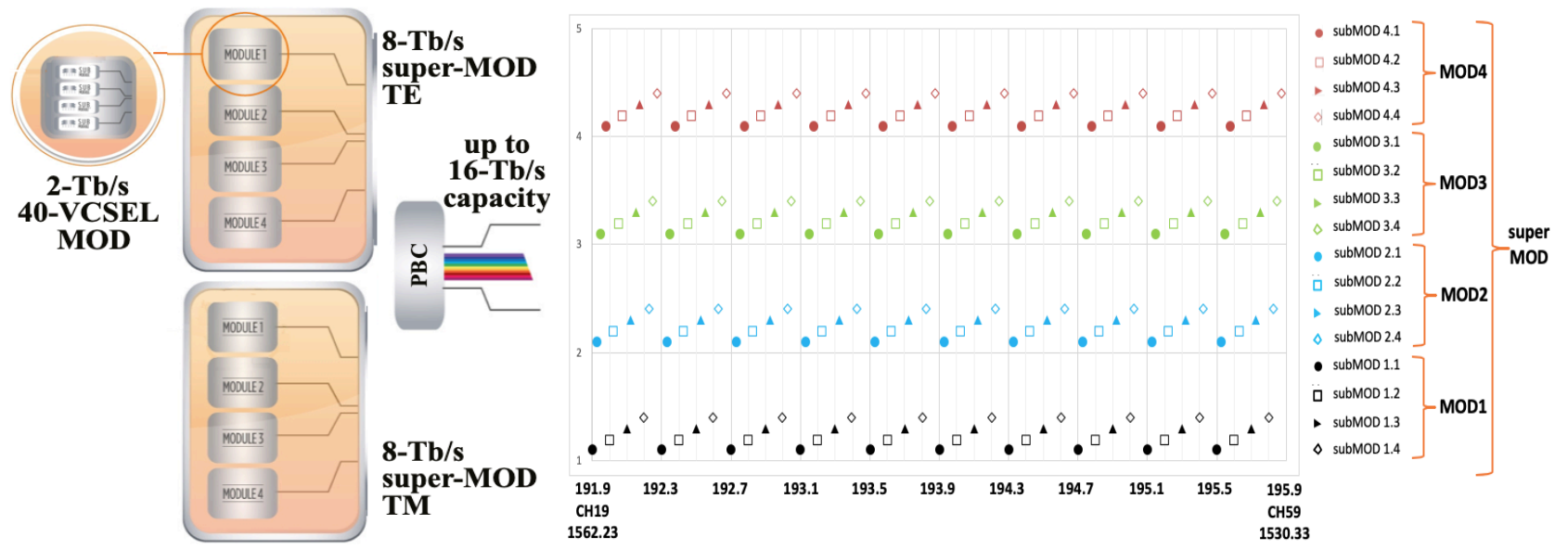

Fig. 5. Left: S-BVT TX structure based on a modular approach. Right: Grid of the VCSEL emission wavelengths for the 4 MODs constituting the super-MOD. 


\section{MAN SWITCHING/AGGREGATION NODE TECHNOLOGIES}

The nodes architecture [24] is designed in order to support the hierarchical network described in Section 2. The HL4 node is based on low-cost switches providing the add/drop operation realized by semiconductor optical amplifier (SOA) wavelength blockers, combined with a demultiplexing AWG and a splitter at the input and a multiplexing AWG and a combiner at the output (Fig. 6 left). The input splitter and the output combiner interface the HL4 with the lower level (HL5) nodes. On the other hand, the HL3 node (shown in Fig. 6 right) presents a photonic switching module (PSM) exploited to drop the incoming traffic or to assign it to the express out path by means of a spatial switch. Aggregate/disaggregate switches allow the routing of the dropped traffic either to the multicast switch (MCS) or to the add switch WSS [25]. For the uplink traffic, a WSS-based add switch is employed to enable contention-less, directionless and colorless add of the traffic to the other HL3 nodes. Switching and routing in space and in spectrum are enabled by this kind of architecture. HL2/HL1 nodes are implemented by the same architecture. The presented node architectures are implemented with the aim to deliver on-chip switch node functionalities, with a modular approach adopted by repeating the same blocks. Both monolithic InP and hybrid WSS of SiPh switches are used, in combination of integrated InP SOAs, while the PSM is based on polymer switches.

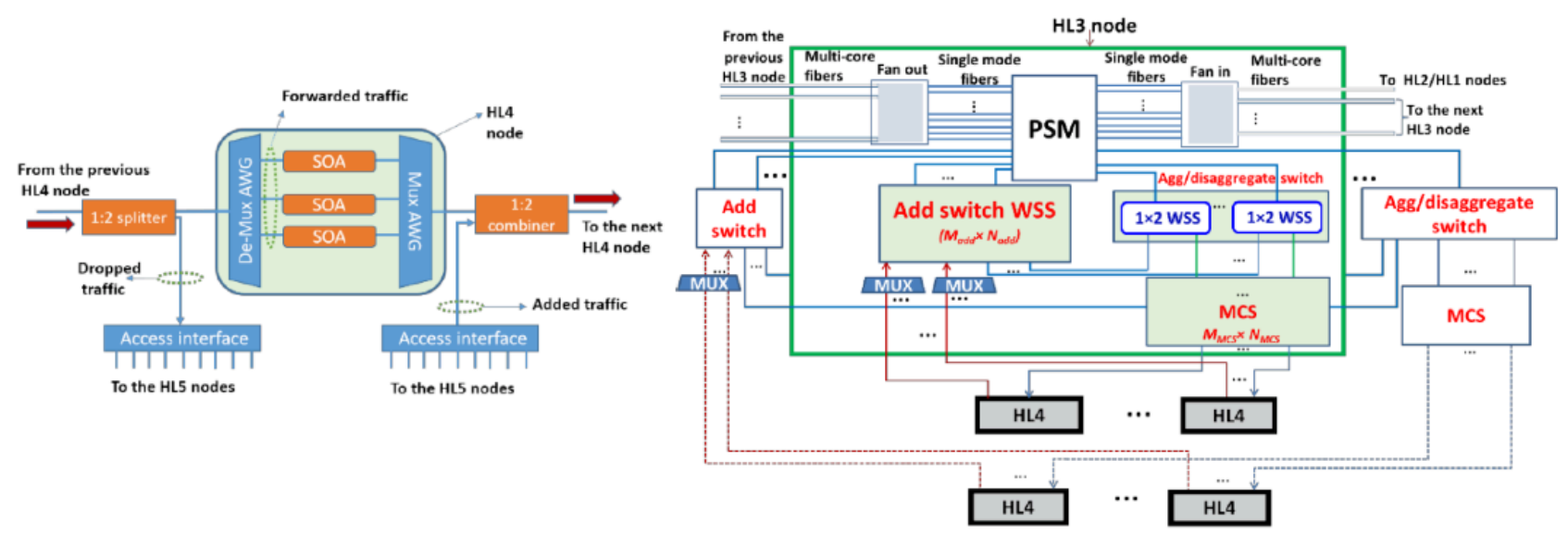

Fig. 6. Left: HL4 node architecture based on SOA switches. Right: HL3 node architecture.

\section{PERFORMANCE EVALUATION}

During propagation in the MAN described so far, the WDM channels generated by the proposed multi-Tb/s VCSEL-based S-BVT are aggregated node by node filling up a 25-GHz WDM grid. Starting from the single-channel performance, we optimized the VCSEL bias current and the modulation amplitude setting them to $8 \mathrm{~mA}$ and $8.5 \mathrm{~mA}$ respectively. Moreover, in order to reduce the inter-channel cross talk [20], we limited the DMT signal electrical occupation to $16 \mathrm{GHz}$, so that the sub-carrier spacing is $62.5 \mathrm{MHz}$. In these conditions, we evaluated the performance of the transmitted channels as a function of the number of nodes crossed along the MAN and for various OSNR, in presence of coherent detection. In particular the presence of MAN nodes is emulated by the cascade of WSS filters: the employed filter transfer function in the simulations is the one measured at the output of a programmable optical filter (Finisar WaveShaper 4000s) encoding the WSS theoretical transfer function of [18]. As previously described, in our architecture at least two WSSs are present: one at the transmitter side (to implement the SSB modulation) and one at the receiver side to demodulate the channel under test. Three 25-GHz spaced VCSELs emitting respectively at $1540 \mathrm{~nm}$ (channel under test), $1539.8 \mathrm{~nm}$ and $1540.2 \mathrm{~nm}$ are employed. The propagation medium is a single-mode fiber, with $0.25 \mathrm{~dB} / \mathrm{km}$ attenuation and $17 \mathrm{ps} / \mathrm{nm} \mathrm{km}$ dispersion at $1550 \mathrm{~nm}$. Erbium-doped fiber amplifiers (EDFAs) with 6-dB noise figure (NF) are used to compensate for the transmission losses.

Figure 7 shows the transmitted capacity as a function of the number of crossed WSSs for three OSNR values. Already for single-channel propagation with an OSNR of $30 \mathrm{~dB}, 50-\mathrm{Gb} / \mathrm{s}$ capacity can be supported only for a single node crossing. The presence of the inter-channel crosstalk reduces the capacity of the 2 WSS case of nearly $30 \%$, whereas for a higher number of filters the reduction is less consistent: in fact, the impairment is caused by the presence of a residual component of the adjacent channel located on the higher frequency subcarriers. It can be noticed that up to 5 WSS can be supported with capacities higher than 50-Gb/s per VCSEL with 25-GHz WDM adjacent channels for OSNR higher than $30 \mathrm{~dB}$, 
whereas the capacities are higher than $40 \mathrm{~Gb} / \mathrm{s}$ for $30 \mathrm{~dB}$ OSNR. Indeed, such OSNR range can guarantee more than 200 $\mathrm{km}$ reach with 35-km spans in the envisaged MAN topology [17].

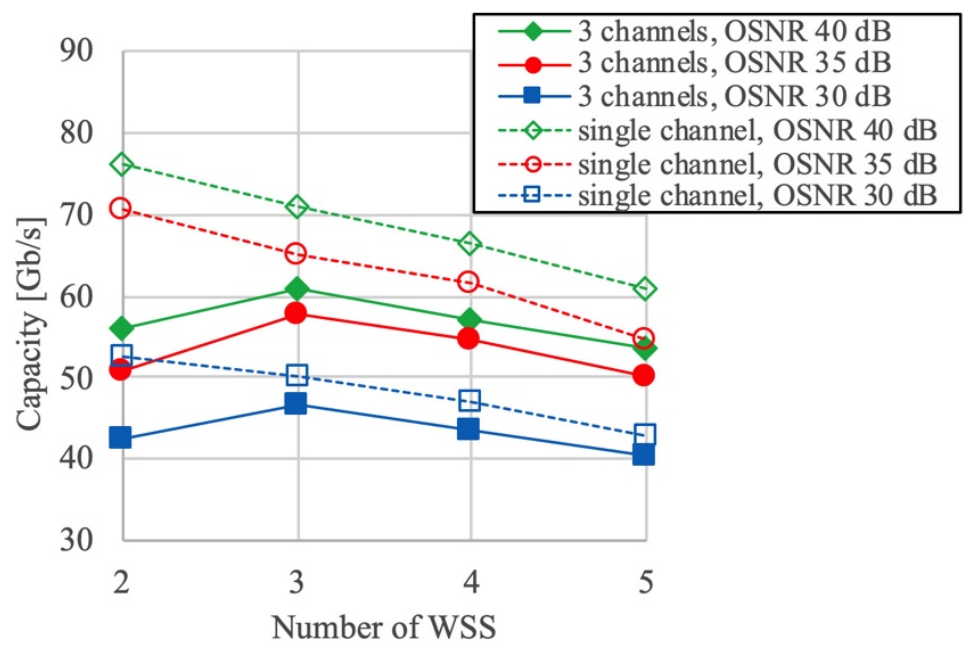

Fig. 7. SSB DMT transmission capacity as a function of number of crossed WSS for different OSNR values ( $40 \mathrm{~dB}$ diamonds, $35 \mathrm{~dB}$ circles, $30 \mathrm{~dB}$ squares): single channel propagation (open symbol and dashed line), 3-channel propagation (full symbol and continuous line)

\section{CONCLUSIONS}

Innovative multi-Tb/s VCSEL-based S-BVT and node structures able to support a flexible and high-capacity MAN have been presented. High-bandwidth long-wavelength multiple VCSELs integrated on a SiPh PIC and on-chip node architecture based on monolithic InP and hybrid technology assure sustainability in terms of cost, power consumption and footprint. Thanks to the adopted modular approach, multi-Tb/s S-BVT operating with 25-GHz granularity. 50-Gb/s SSB DMT modulation per VCSEL has been experimentally demonstrated, supported by CO-Rx in order to face also hundreds of $\mathrm{km}$ reaches with a reduced DSP complexity. This rate per VCSEL has been also verified by simulation in case of multichannel propagation in presence of multiple nodes crossing. The developed photonic technological solutions allow to face the request of a huge throughput and traffic scaling of the future MAN matching the performance requirements.

\section{ACKNOWLEDGMENT}

This paper is part of a project that has received funding from the European Union's Horizon 2020 research and innovation programme under grant agreement PASSION No 780326.

\section{REFERENCES}

[1] M. Svaluto Moreolo et al., " Spectrum/Space Switching and Multi-Terabit Transmission in Agile Optical Metro Networks," in Proc. OECC/PSC 2019, paper C000235, July 2019.

[2] H. Adams, "Trends in Metro Optical Networks," www.ihsmarkit.com (2018)

[3] J. Lin, et al. "Joint Digital and Optical Pre-compensation for $720 \mathrm{~Gb} / \mathrm{s}$ All-Silicon IQ modulator Single Carrier Transmission", Proc. ECOC 2018, paper We3G.2, September 2018.

[4] J. Renaudier, "100nm ultra-wide optical fiber transmission systems using semiconductor optical amplifiers", Proc. ECOC 2018, paper Mo4G.5 September 2018.

[5] www.passion-project.eu 
[6] A. Gatto et al., "VCSEL-based communications for metro and access networks," in Proc. OECC/PSC 2018, paper 8751367, September 2018.

[7] A. Gatto et al., "Discrete multitone modulation for short-reach mode division multiplexing transmission," Journal of Lightwave Technology, 37, 20, 5185-5192 (2019).

[8] M. Svaluto Moreolo et al., "Modular SDN-enabled S-BVT Adopting Widely Tunable MEMS VCSEL for Flexible/Elastic Optical Metro Networks," in Proc. OFC 2018, paper M1A.7, March 2018.

[9] D. Larrabeiti et al., "All-optical paths across multiple hierarchical levels in large metropolitan area networks," Proc. ACP 2019, November 2019.

[10]A. Boletti, et al., "Performance analysis of communication links based on VCSEL and silicon photonics technology for high-capacity data-intensive scenario", Opt. Express, 23, 1806-1815 (2015).

[11] C. Xie et al., "400-Gb/s PDM-4PAM WDM System Using a Monolithic $2 \times 4$ VCSEL Array and Coherent Detection," J. Lightwave Technol., 33, (3), pp. 670-677, 2015.

[12] A. Gatto et al., "Very high-capacity short-reach VCSEL systems exploiting multicarrier intensity modulation and direct detection" Opt. Express, 24, 12, 12769-12775 (2016)

[13] A. Gatto et al., "Beyond $50 \mathrm{~Gb} / \mathrm{s}$ directly-modulated long-wavelength VCSELs for next-gen access network," Proc. IEEE Photonics Society Summer Topical Meeting, 8795016, July 2019.

[14] A. Gatto, et al., "FDM and DMT performance comparison in high capacity point-to-point fibre links for intra/inter-datacentre connections," Optical Fiber Technology, 42, 92-96 (2018).

[15] C. Xie, et al., "Single VCSEL 100-Gb/s short reach system using discrete multi-tone modulation and direct detection," in Proc. OFC 2015, paper Tu2H.2, March 2015.

[16] M. Svaluto Moreolo, "Exploring the Potential of VCSEL Technology for Agile and High Capacity Optical Metro Networks," in Proc. 22nd Conference on Optical Network Design and Modelling - ONDM 2018, May 2018.

[17] M. Rapisarda, et al. "Impact of Chirp in High-Capacity Optical Metro Networks Employing Directly-Modulated VCSELs," Photonics, 5, (4), pp. 51, 2018.

[18] C. Pulikkaseril et al., "Spectral modeling of channel band shapes in wavelength selective switches." Opt. Express, 19, 8458-8470, 2011.

[19]P. S. Chow et al., "A practical discrete multitone transceiver loading algorithm for data transmission over spectrally shaped channels," IEEE Trans. on Comm., 43, 773-775, 1995.

[20]P. Parolari et al., "Effect of filtering in dense WDM metro networks adopting VCSEL-based multi-Tb/s transmitters," Proc. ICTON 2019, July 2019.

[21] S. Bhat, et al., "Low Loss Devices fabricated on the Open Access 3mm SOI Waveguide Platform at VTT" in Proc. ECIO 2019, paper T.Po2.18, 2019.

[22] A. Gatto et al., "Disruptive photonic technologies for the future sustainable high-capacity metro network," Proc. ICTON 2019, July 2019.

[23] M. Svaluto Moreolo et al., "VCSEL-based sliceable bandwidth/bitrate variable transceivers," in Proc. SPIE, 10946, 2019, 1094606, Metro and Data Center Optical Networks and Short-Reach Links II, February 2019.

[24] N. Calabretta et al., "Photonic integrated WDM cross-connects for optical metro and data center networks," in Proc. SPIE, 10945, 2019, 1094507, Broadband Access Communication Technologies XIII, February 2019.

[25] N. Tessema et al., "A novel modular ROADM node with traffic aggregation/disaggregation for ultra-high capacity SDM metro networks,” in Proc. ECOC 2018, 8535441, September 2018. 